\title{
The Software Reliability Growth Models for Software Life-Cycle Based on NHPP
}

\author{
Kyung H. Nam ${ }^{1}$. Do Hoon Kim ${ }^{2}$ \\ ${ }^{1}$ Department of Applied Information Statistics, Kyonggi University \\ ${ }^{2}$ Department of Applied Information Statistics, Kyonggi University
}

(Received July 2009; accepted May 2010)

\begin{abstract}
This paper considers the differences in the software execution environments in the testing phase and the operational phase to determine the optimal release time and warranty period of software systems. We formulate equations for the total expected software cost until the end of the software life cycle based on the NHPP. In addition, we derive the optimal release time that minimizes the total expected software cost for an imperfect debugging software reliability model. Finally, we analyze the sensitivity of the optimal testing and maintenance design related to variation of the cost model parameters based on the fault data observed in the actual testing process, and discuss the quantitative properties of the proposed model.
\end{abstract}

Keywords: Software reliability growth model, nonhomogeneous Poisson process, software release time, warranty period, software maintenance activity.

\section{Introduction}

Every software project manager is expected to release fault-free computer software to his/her customers. Due to the complicity of current software, it has been increasingly difficult for development managers to produce highly reliable and effective software systems. In the context of the software development, penalty costs for software failures are even more significant. As a result, the determination of the software release time is an important factor in the success or failure of software. Okumoto and Goel (1980) assumed that the number of software faults detected in the software testing phase conforms to an exponential software reliability model (Goel and Okumoto, 1979) based on the NHPP and derived the optimal release time minimizing the total expected cost. Koch and Kubat (1983) assumed the Jelinski and Moranda (1972) model for the software fault detection process and discussed a similar problem.

It is not easy to prevent the occurrence of failures caused by software faults after release since detecting and removing all of the faults latent in the software in the actual testing process are extremely difficult. In many cases, the development manager must investigate the causes of software

This work was supported by Kyonggi University Research Grant 2007.

${ }^{1}$ Corresponding author: Professor, Department of Applied Information Statistics, Kyonggi University, Suwon 443-760, Korea. E-mail: knam@kyonggi.ac.kr 
failures that occurred after the software release based on the maintenance and warranty contract with the user to detect and remove the faults. To perform maintenance in the operational phase (after the release), the software development manager is requested to reduce the management costs in the operational phase and effectively utilize the human resources in contrast that require continued support by the development project team. Although the length of period for continued maintenance by the development project team has the same importance as the release time, but they have been considered in the little literature.

Yamada (1994) and Kimura et al. (1999) considered the problem of determining the optimal release time while assuming the software warranty period to be a random variable. Pham and Zhang (1999) proposed a software cost model that simultaneously considered the warranty and the risk after release. However, the operational maintenance of the software is provided as a service after release by the development manager and must be designed by the maintenance contract itself and the product delivery date. Dohi et al. (2000) and Rinsaka and Sandoh (1999) determine the optimal warranty period that minimizes the total expected software cost based on the assumption that the debugging process in the testing process is described by an NHPP. However, most of the research does not fully consider the differences in the debugging environments in the testing phase and the operational phase, and present their discussions based on a simple cost structure.

This paper proposes a model for the differences in the software execution environments in the testing phase and the operational phase. We consider that the actual maintenance activities are ineffective during the warranty period. We formulate the mathematical equations for the total expected software cost until the end of the software life cycle based on the NHPP. In addition, we derive the optimal release time and warranty period that minimizes the total expected software cost for an imperfect debugging software reliability model. Finally, we analyze the sensitivity of the optimal testing and maintenance design related to the variation of the cost model parameters based on the fault data observed in the actual testing process, and discuss the quantitative properties of the proposed model.

\section{Model Descriptions}

\subsection{Assumptions}

We consider two periodic software maintenance policies in association with an imperfect debugging software reliability growth model. We construct the several costs required in the software development process for failure-occurrence time data to formulate the total expected software cost. The failure-occurrence time is the time-interval between software failure-occurrences. Throughout this paper, we postulate the following assumptions.

Assumption 2.1. The software system starts to test at time $t=0$.

Assumption 2.2. Software development manager conducts the maintenance activities(e.g. patch, update) during the warranty period $t_{w}$ after the software release.

Assumption 2.3. The periodic software maintenance is done at periodic time $k t_{w} / N,(k=1,2, \ldots, N$, $\left.t_{w} \geq 0\right)$, and is stopped the maintenance activities at the $N^{\text {th }}$ periodic software maintenance.

Assumption 2.4. The life cycle $t_{L}>0$ of a software product is known.

Assumption 2.5. A new fault may be introduced into the software system due to an imperfect debugging. 
Let $\{N(t), t \geq 0\}$ be a counting process representing the cumulative number of software faults detected or removed up to time $t$. The detection time of each software fault can be formulated as an NHPP as follows:

$$
\operatorname{Pr}\{N(t)=n\}=\frac{\{m(t)\}^{n}}{n !} \exp \{-m(t)\}, \quad(n=0,1,2, \ldots),
$$

where $m(t)$ is the mean value function of the NHPP, that is, the expected cumulative number of faults detected up to time $t$.

Pham (1993) introduced an NHPP SRGM that is subject to an imperfect debugging. He assumed that the detected faults are removed and then there is a possibility to introduce new faults with constant rate $\beta$. Let $a(t)$ be the number of faults to be eventually detected, where $a(t)$ is the time-dependent fault content function. The mean value function $m(t)$ can be given as the solution of the following system of differential equations:

$$
\begin{aligned}
\frac{\partial m(t)}{\partial t} & =b[a(t)-m(t)], \quad \frac{\partial a(t)}{\partial t}=\beta \frac{\partial m(t)}{\partial t}, \\
a(0) & =a, m(0)=0,
\end{aligned}
$$

where $a$ is the number of faults to be eventually detected. Solving the Equation (2.2), we obtain the mean value function $m(t)$ and instantaneous fault detection rate $\lambda(t)$ as follows:

$$
\begin{aligned}
m(t) & =\frac{a}{1-\beta}\left[1-e^{-(1-\beta) b t}\right], \\
\lambda(t) & =a b e^{-(1-\beta) b t} .
\end{aligned}
$$

To determine the optimal release time and warranty period of software systems, we propose two periodic software maintenance policies in terms of the behavior of instantaneous fault detection rate with an imperfect debugging SRGM.

Case 1. Let us assume that after the software release, the software reliability growth occurs(assuming that we correct only major faults that will improve the reliability of the software). That is, the instantaneous fault detection rate after the release time assumes the decreasing function.

Case 2. Let us assume that during the warranty period, the software reliability growth does not occur(assuming that we correct only minor faults). That is, the instantaneous fault detection rate after the release follows that of the release time point, is continued as constant.

In Figure 2.1, $t_{0}$ denotes the software release time, $t_{w}$ denotes the software warranty period, $t_{L}$ denotes the software life cycle and $N$ denotes the number of maintenance activities during the warranty period and $0<t_{1}\left(=t_{0}+t_{w} / N\right)<t_{2}\left(=t_{0}+2 t_{w} / N\right)<\cdots<t_{N}\left(=t_{0}+t_{w}\right)$.

The instantaneous fault detection rates of the proposed periodic maintenance policy are as follows.

1. Policy 1 based on Case(1)

$$
\lambda_{p m}(t)=\lambda(t), \quad 0<t \leq t_{0}+t_{L} .
$$

2. Policy 2 based on Case(2)

$$
\lambda_{p m}(t)= \begin{cases}\lambda(t), & 0<t \leq t_{0} \\ \lambda\left(t_{0}\right), & t_{0}<t \leq t_{0}+t_{L}\end{cases}
$$




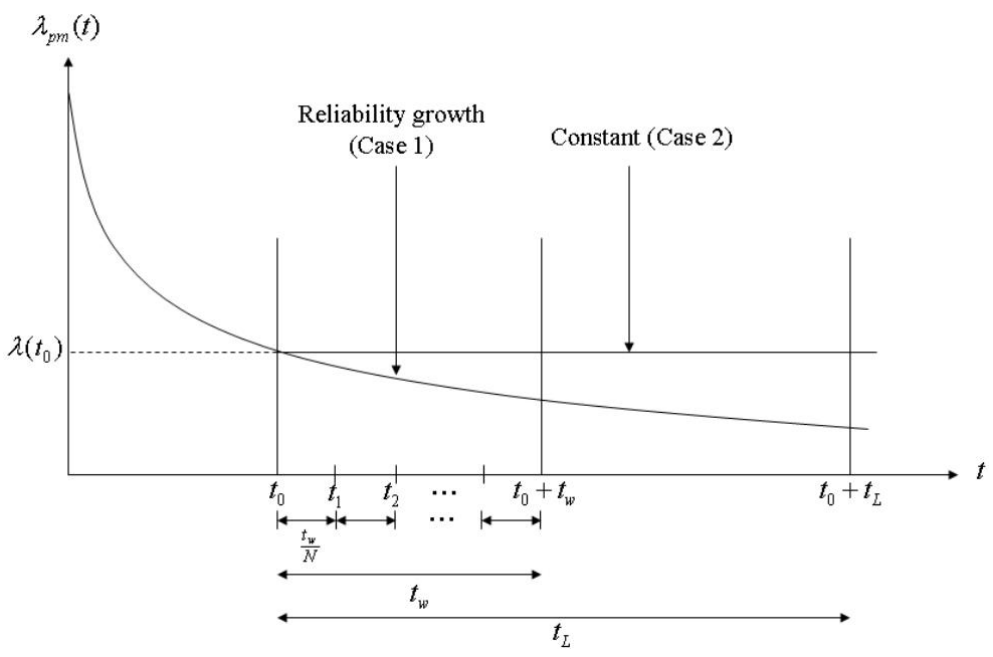

Figure 2.1. Software reliability growth during the warranty period of ineffective maintenance policies

\subsection{Total expected software cost}

To formulate the total expected software cost based on data related to the number of faults detected in the software development process, the cost parameters are defined as follows.

$$
\begin{aligned}
& c_{0}(>0) \text { : cost to remove each fault in the testing phase. } \\
& c_{w}(>0) \text { : cost to remove each fault during the warranty period. } \\
& c_{L}(>0) \text { : cost to remove each fault after the warranty period. } \\
& c_{p}(>0) \text { : unit maintenance cost. } \\
& c_{t}(>0) \text { : testing cost per unit time. }
\end{aligned}
$$

Based on the Pham (1993)'s NHPP model described in Section 2.1, we formulate the total expected software cost in the software development project.

Several expected costs that are required during the software development project can be determined as follows:

1) The expected cumulative number of software faults removed in the testing phase $\left(0, t_{0}\right)$ is given by

$$
E\left[N\left(t_{0}\right)\right]=m\left(t_{0}\right),
$$

where $N\left(t_{0}\right)$ is the number of faults detected up to time $t_{0}$. Hence, the expected cost to remove all faults detected by time $t_{0}, \mathrm{EC}_{0}\left(t_{0}, t_{w}\right)$, can be expressed as

$$
\mathrm{EC}_{0}\left(t_{0}, t_{w}\right)=c_{0} \cdot E\left[N\left(t_{0}\right)\right]=c_{0} m\left(t_{0}\right) .
$$

2) The expected cumulative number of software faults removed during warranty period $\left[t_{0}, t_{0}+t_{w}\right)$ is given by

$$
E\left[N\left(t_{0}+t_{w}\right)-N\left(t_{0}\right)\right]=\left[m\left(t_{0}+t_{w}\right)-m\left(t_{0}\right)\right] .
$$


Hence, the expected cost to remove all faults detected by time $\left[t_{0}, t_{0}+t_{w}\right), \mathrm{EC}_{w}\left(t_{0}, t_{w}\right)$, can be expressed as

$$
\mathrm{EC}_{w}\left(t_{0}, t_{w}\right)=c_{w} \cdot E\left[N\left(t_{0}+t_{w}\right)-N\left(t_{0}\right)\right]=c_{w}\left[m\left(t_{0}+t_{w}\right)-m\left(t_{0}\right)\right]
$$

3) The expected cumulative number of software faults removed after warranty period $\left[t_{0}+t_{w}, t_{0}+\right.$ $\left.t_{L}\right)$ is given by

$$
E\left[N\left(t_{0}+t_{L}\right)-N\left(t_{0}+t_{w}\right)\right]=\left[m\left(t_{0}+t_{L}\right)-m\left(t_{0}+t_{w}\right)\right] .
$$

Hence, the expected cost to remove all faults detected by time $\left[t_{0}+t_{w}, t_{0}+t_{L}\right), \mathrm{EC}_{L}\left(t_{0}, t_{w}\right)$, can be expressed as

$$
\mathrm{EC}_{L}\left(t_{0}, t_{w}\right)=c_{L} \cdot E\left[N\left(t_{0}+t_{L}\right)-N\left(t_{0}+t_{w}\right)\right]=c_{L}\left[m\left(t_{0}+t_{L}\right)-m\left(t_{0}+t_{w}\right)\right] .
$$

4) Cost to do patch, $\mathrm{EC}_{p}\left(t_{0}, t_{w}\right)$ is proportional to the number of maintenance activities

$$
\mathrm{EC}_{p}\left(t_{0}, t_{w}\right)=c_{p} \cdot N
$$

5) Testing cost, $\mathrm{EC}_{t}\left(t_{0}, t_{w}\right)$ is a linear function of time $t_{0}$ and $t_{w}$

$$
\mathrm{EC}_{t}\left(t_{0}, t_{w}\right)=c_{t}\left(t_{0}+t_{w}\right)
$$

Therefore, the total expected software cost, $\operatorname{EC}\left(t_{0}, t_{w}\right)$ can be expressed as

$$
\begin{aligned}
\mathrm{EC}\left(t_{0}, t_{w}\right)= & c_{0} m\left(t_{0}\right)+c_{w}\left[m\left(t_{0}+t_{w}\right)-m\left(t_{0}\right)\right] \\
& +c_{L}\left[m\left(t_{0}+t_{L}\right)-m\left(t_{0}+t_{w}\right)\right]+c_{p} N+c_{t}\left(t_{0}+t_{w}\right) .
\end{aligned}
$$

By the total expected software cost of proposed maintenance policies in Case 1 and 2 replacing $\lambda(t)$ with $a b e^{-(1-\beta) b t}$ in Equation (2.3), we can be calculated as follows.

1. Policy 1 based on Case(1)

$$
\begin{aligned}
\mathrm{EC}_{1}\left(t_{0}, t_{w}\right)= & c_{0} \int_{0}^{t_{0}} a b e^{-(1-\beta) b t} d t+c_{w} \int_{t_{0}}^{t_{0}+t_{w}} a b e^{-(1-\beta) b t} d t+c_{L} \int_{t_{0}+t_{w}}^{t_{0}+t_{L}} a b e^{-(1-\beta) b t} d t \\
& +c_{p} N+c_{t}\left(t_{0}+t_{w}\right)
\end{aligned}
$$

2. Policy 2 based on Case(2)

$$
\begin{aligned}
\mathrm{EC}_{2}\left(t_{0}, t_{w}\right)= & c_{0} \int_{0}^{t_{0}} a b e^{-(1-\beta) b t} d t+c_{w} \int_{t_{0}}^{t_{0}+t_{w}} a b e^{-(1-\beta) b t_{0}} d t+c_{L} \int_{t_{0}+t_{w}}^{t_{0}+t_{L}} a b e^{-(1-\beta) b t_{0}} d t \\
& +c_{p} N+c_{t}\left(t_{0}+t_{w}\right) .
\end{aligned}
$$

In the next chapter, we discuss the optimal release time $t_{0}^{*}$ and the optimal warranty period $t_{w}^{*}$ minimizing the total expected software cost.

\section{Optimal Software Release Time and Warranty Period}

We now derive the optimal release time and the optimal warranty period for proposed policies that minimize the total expected software cost, $\mathrm{EC}_{1}\left(t_{0}, t_{w}\right), \mathrm{EC}_{2}\left(t_{0}, t_{w}\right)$, respectively. 


\subsection{Policy 1 based on Case(1)}

To show the existence and uniqueness of the optimal release time $t_{0}^{*}$ and the optimal warranty period $t_{w}^{*}$, which minimize $\mathrm{EC}_{1}\left(t_{0}, t_{w}\right)$, we rewrite the total expected software cost of Equation (2.15) as follows.

$$
\begin{aligned}
\mathrm{EC}_{1}\left(t_{0}, t_{w}\right)= & \frac{a c_{0}}{1-\beta}\left[1-e^{-(1-\beta) b t_{0}}\right]+\frac{a c_{w}}{1-\beta}\left[e^{-(1-\beta) b t_{0}}\left(1-e^{-(1-\beta) b t_{w}}\right)\right] \\
& +\frac{a c_{L}}{1-\beta}\left[e^{-(1-\beta) b t_{0}}\left(e^{-(1-\beta) b t_{w}}-e^{-(1-\beta) b t_{L}}\right)\right]+c_{p} N+c_{t}\left(t_{0}+t_{w}\right) .
\end{aligned}
$$

The following assumptions are set:

$(\mathrm{A}-\mathrm{I}) c_{L}>c_{w}>c_{0}$

(A-II) $c_{w}\left(1-e^{-(1-\beta) b t_{L}}\right)>c_{0}$

(A-III) $c_{w}\left(1-e^{-(1-\beta) b t_{w}}\right)+c_{L}\left(e^{-(1-\beta) b t_{w}}-e^{-(1-\beta) b t_{L}}\right)>c_{0}$

and let

$$
Q_{1}\left(t_{w}\right)=a b\left[c_{0}-c_{w}\left(1-e^{-(1-\beta) b t_{w}}\right)-c_{L}\left(e^{-(1-\beta) b t_{w}}-e^{-(1-\beta) b t_{L}}\right)\right]+c_{0} .
$$

Theorem 3.1. Given $c_{0}, c_{w}, c_{L}, c_{p}, c_{t}, N, t_{w}, t_{L}$, the optimal release time $t_{0}^{*}$, which minimizes the total expected software cost, $E C_{1}\left(t_{0}, t_{w}\right)$ can be determined as follows based on A-I to A-III.

Case 1. When $Q_{1}\left(t_{w}\right)<0$, a finite and unique $t_{0}^{*}(>0)$ that minimizes the total expected software cost $E C_{1}\left(t_{0}, t_{w}\right)$ exists:

$$
\begin{aligned}
E C_{1}\left(t_{0}^{*}, t_{w}\right)= & \frac{a c_{0}}{1-\beta}\left[1-e^{-(1-\beta) b t_{0}^{*}}\right]+\frac{a c_{w}}{1-\beta}\left[e^{-(1-\beta) b t_{0}^{*}}\left(1-e^{-(1-\beta) b t_{w}}\right)\right] \\
& +\frac{a c_{L}}{1-\beta}\left[e^{-(1-\beta) b t_{0}^{*}}\left(e^{-(1-\beta) b t_{w}}-e^{-(1-\beta) b t_{L}}\right)\right]+c_{p} N+c_{t}\left(t_{0}^{*}+t_{w}\right) .
\end{aligned}
$$

Case 2. When $Q_{1}\left(t_{w}\right)>0, t_{0}^{*}=0$ and

$$
E C_{1}\left(t_{0}^{*}, t_{w}\right)=\frac{a c_{w}}{1-\beta}\left[1-e^{-(1-\beta) b t_{w}}\right]+\frac{a c_{L}}{1-\beta}\left[e^{-(1-\beta) b t_{w}}-e^{-(1-\beta) b t_{L}}\right]+c_{p} N+c_{t} t_{w} .
$$

The software debugging becomes optimal by only an acceptance test by the user in the operational phase.

Proof.

$$
\begin{aligned}
\frac{\partial \mathrm{EC}_{1}\left(t_{0}, t_{w}\right)}{\partial t_{0}}= & a b c_{0} e^{-(1-\beta) b t_{0}}-a b c_{w} e^{-(1-\beta) b t_{0}}\left(1-e^{-(1-\beta) b t_{w}}\right) \\
& -a b c_{L} e^{-(1-\beta) b t_{0}}\left(e^{-(1-\beta) b t_{w}}-e^{-(1-\beta) b t_{L}}\right)+c_{t} .
\end{aligned}
$$

Let $\delta_{1}\left(t_{0}, t_{w}\right)$ denote the right-hand side of Equation (3.5), then

$$
\delta_{1}\left(0, t_{w}\right)=a b\left[c_{0}-c_{w}\left(1-e^{-(1-\beta) b t_{w}}\right)-c_{L}\left(e^{-(1-\beta) b t_{w}}-e^{-(1-\beta) b t_{L}}\right)\right]+c_{t}
$$

and

$$
\lim _{t_{0} \rightarrow+\infty} \delta_{1}\left(t_{0}, t_{w}\right)=c_{t}
$$


Differentiating Equation (3.5) with respect to $t_{0}$, we obtain

$$
\frac{\partial^{2} \mathrm{EC}_{1}\left(t_{0}, t_{w}\right)}{\partial^{2} t_{0}}=a b^{2}(1-\beta) e^{-(1-\beta) b t_{0}}\left[-c_{0}+c_{w}\left(1-e^{-(1-\beta) b t_{w}}\right)+c_{L}\left(e^{-(1-\beta) b t_{w}}-e^{-(1-\beta) b t_{L}}\right)\right] .
$$

Letting $\gamma_{1}\left(t_{0}, t_{w}\right)$ denote the right-hand side of Equation (3.8),

$$
\gamma_{1}\left(0, t_{w}\right)=a b^{2}(1-\beta)\left[-c_{0}+c_{w}\left(1-e^{-(1-\beta) b t_{w}}\right)+c_{L}\left(e^{-(1-\beta) b t_{w}}-e^{-(1-\beta) b t_{L}}\right)\right] .
$$

Letting $A_{1}\left(t_{w}\right)$ denote the right-hand side of Equation (3.9), $A_{1}(0)>0$ is equivalent to $c_{L}(1-$ $\left.e^{-(1-\beta) b t_{L}}\right)>c_{0}, A_{1}\left(t_{L}\right)>0$ is equivalent to $c_{w}\left(1-e^{-(1-\beta) b t_{L}}\right)>c_{0}$. Therefore, from assumption A-I, $A_{1}(0)>A_{1}\left(t_{L}\right)$ holds. Furthermore, $A_{1}^{\prime}\left(t_{w}\right)$ becomes

$$
A_{1}^{\prime}\left(t_{w}\right)=-\left(c_{L}-c_{w}\right) a b^{3}(1-\beta)^{2} e^{-(1-\beta) b t_{w}}<0,
$$

$\gamma_{1}\left(0, t_{w}\right)>0$. Next

$$
\lim _{t_{0} \rightarrow+\infty} \gamma_{1}\left(t_{0}, t_{w}\right)=0
$$

$\partial \gamma_{1}\left(t_{0}, t_{w}\right) / \partial t_{0}<0$ is equivalent to

$$
c_{w}\left(1-e^{-(1-\beta) b t_{w}}\right)+c_{L}\left(e^{-(1-\beta) b t_{w}}-e^{(1-\beta) b t_{L}}\right)>c_{0} .
$$

Theorem 3.2. Given $c_{0}, c_{w}, c_{L}, c_{p}, c_{t}, N, t_{0}, t_{L}$, the optimal warranty period $t_{w}^{*}$, which minimizes the total expected software cost $E C_{1}\left(t_{0}, t_{w}\right)$ based on $A-I$ is determined by the following.

Case 1. When $c_{t} \geq a b e^{-(1-\beta) b t_{0}}\left(c_{L}-c_{w}\right), t_{w}^{*}=0$ minimizes $E C_{1}\left(t_{0}, t_{w}\right)$.

$$
E C_{1}\left(t_{0}, t_{w}^{*}\right)=\frac{a c_{0}}{1-\beta}\left[1-e^{-(1-\beta) b t_{0}}\right]+\frac{a c_{L}}{1-\beta}\left[e^{-(1-\beta) b t_{0}}\left(1-e^{-(1-\beta) b t_{L}}\right)\right]+c_{p} N+c_{t} t_{0}
$$

Case 2. When $c_{t}<a b e^{-(1-\beta) b t_{0}}\left(c_{L}-c_{w}\right)$ and $c_{t}>a b e^{-(1-\beta) b\left(t_{0}+t_{L}\right)}\left(c_{L}-c_{w}\right)$, a finite and unique $t_{w}^{*}\left(0<t_{w}<t_{L}\right)$ that minimizes the total expected software cost $E C_{1}\left(t_{0}, t_{w}\right)$ exists.

$$
\begin{aligned}
E C_{1}\left(t_{0}, t_{w}^{*}\right)= & \frac{a c_{0}}{1-\beta}\left[1-e^{-(1-\beta) b t_{0}}\right]+\frac{a c_{w}}{1-\beta}\left[e^{-(1-\beta) b t_{0}}\left(1-e^{-(1-\beta) b t_{w}^{*}}\right)\right] \\
& +\frac{a c_{L}}{1-\beta}\left[e^{-(1-\beta) b t_{0}}\left(e^{-(1-\beta) b t_{w}^{*}}-e^{-(1-\beta) b t_{L}}\right)\right]+c_{p} N+c_{t}\left(t_{0}+t_{w}^{*}\right)
\end{aligned}
$$

Case 3. When $c_{t} \leq a b e^{-(1-\beta) b\left(t_{0}+t_{L}\right)}\left(c_{L}-c_{w}\right), t_{w}^{*}=t_{L}$ minimizes $E C_{1}\left(t_{0}, t_{w}\right)$.

$$
\begin{aligned}
E C_{1}\left(t_{0}, t_{w}^{*}\right)= & \frac{a c_{0}}{1-\beta}\left[1-e^{-(1-\beta) b t_{0}}\right]+\frac{a c_{w}}{1-\beta}\left[e^{-(1-\beta) b t_{0}}\left(1-e^{-(1-\beta) b t_{L}}\right)\right] \\
& +c_{p} N+c_{t}\left(t_{0}+t_{L}\right) .
\end{aligned}
$$

Proof.

$$
\frac{\partial \mathrm{EC}_{1}\left(t_{0}, t_{w}\right)}{\partial t_{w}}=a b e^{-(1-\beta) b\left(t_{0}+t_{w}\right)}\left(c_{w}-c_{L}\right)+c_{t} .
$$

Let $\xi_{1}\left(t_{0}, t_{w}\right)$ denote the right-hand side of Equation (3.16). Then

$$
\xi_{1}\left(t_{0}, 0\right)=a b e^{-(1-\beta) b t_{0}}\left(c_{w}-c_{L}\right)+c_{t}
$$


and

$$
\lim _{t_{w} \rightarrow+\infty} \xi_{1}\left(t_{0}, t_{w}\right)=c_{t} .
$$

Differentiating Equation (3.17) with respect to $t_{w}$, we obtain

$$
\frac{\partial \xi_{1}\left(t_{0}, t_{w}\right)}{\partial t_{w}}=a b^{2}(1-\beta) e^{-(1-\beta) b\left(t_{0}+t_{w}\right)}\left(c_{L}-c_{w}\right)
$$

Let $\eta_{1}\left(t_{0}, t_{w}\right)$ denote the right-hand side of Equation (3.16). According to the assumption A-I, $\eta_{1}\left(t_{0}, t_{w}\right)>0$.

Now, we introduce the algorithm presented by Rinsaka and Dohi (2006) for simultaneously determining the optimal release time and the optimal warranty period which minimizes $\mathrm{EC}_{1}\left(t_{0}, t_{w}\right)$ as discussed in maintenance policy of hardware product (Mi, 1994; Cha, 2000, 2001).

$\underline{\text { Algorithm }}$

Step 1. If $\left(t_{0}, t_{w}\right)$ which satisfied (3.20) exists for $t_{0} \geq 0$ and $0 \leq t_{w} \leq t_{L}$, go to Step 2. If not, execute Step 3.

$$
\frac{\partial \mathrm{EC}_{1}\left(t_{0}, t_{w}\right)}{\partial t_{0}}=\frac{\partial \mathrm{EC}_{1}\left(t_{0}, t_{w}\right)}{\partial t_{w}}=0
$$

Step 2. The Hessian is defined as

$$
H\left(t_{0}, t_{w}\right)=\frac{\partial^{2} \mathrm{EC}_{1}\left(t_{0}, t_{w}\right)}{\partial t_{0}^{2}} \frac{\partial^{2} \mathrm{EC}_{1}\left(t_{0}, t_{w}\right)}{\partial t_{w}^{2}}-\left(\frac{\partial^{2} E C_{1}\left(t_{0}, t_{w}\right)}{\partial t_{0} \partial t_{w}}\right)^{2} .
$$

If $H\left(t_{0}, t_{w}\right)>0$ and $\partial^{2} \mathrm{EC}_{1}\left(t_{0}, t_{w}\right) / \partial t_{0}^{2}>0$, the solution found in Step 1 becomes the joint optimal policy $\left(t_{0}^{* *}, t_{w}^{* *}\right)$, and the algorithm ends. If not, execute Step 3.

Step 3. Determine $t_{w}^{*}\left(0 \leq t_{w}^{*} \leq t_{L}\right)$ that satisfies $\partial \mathrm{EC}_{1}\left(0, t_{w}\right) / \partial t_{w}=0$, and set

$$
\mathrm{EC}_{1}^{(1)}\left(t_{0}^{*}, t_{w}^{*}\right)=\mathrm{EC}_{1}\left(0, t_{w}^{*}\right)
$$

Step 4. Determine $t_{0}^{*}\left(0 \leq t_{0}^{*}<\infty\right)$ that satisfies $\partial \mathrm{EC}_{1}\left(t_{0}, 0\right) / \partial t_{0}=0$, and set

$$
\mathrm{EC}_{1}^{(2)}\left(t_{0}^{*}, t_{w}^{*}\right)=\mathrm{EC}_{1}\left(t_{0}^{*}, 0\right) \text {. }
$$

Step 5. Determine $t_{0}^{*}\left(0 \leq t_{0}^{*}<\infty\right)$ that satisfies $\partial \mathrm{EC}_{1}\left(t_{0}, t_{L}\right) / \partial t_{0}=0$, and set

$$
\mathrm{EC}_{1}^{(3)}\left(t_{0}^{*}, t_{w}^{*}\right)=\mathrm{EC}_{1}\left(t_{0}^{*}, t_{L}^{*}\right)
$$

Step 6. The minimum total expected software cost is obtained from $\mathrm{EC}_{1}\left(t_{0}^{* *}, t_{w}^{* *}\right)=\min _{i=1,2,3} \mathrm{EC}_{1}^{(i)}$ $\left(t_{0}^{*}, t_{w}^{*}\right)$ and the corresponding $\left(t_{0}^{* *}, t_{w}^{* *}\right)$ becomes the joint optimal policy.

\subsection{Policy 2 based on Case(2)}

By utilizing the similar technique as in Policy 1, we find the optimal release time $t_{0}^{*}$ and the optimal warranty period $t_{w}^{*}$, which minimize $\mathrm{EC}_{2}\left(t_{0}, t_{w}\right)$. Rewriting the total expected software cost of 
Equation (2.16), we have

$$
\mathrm{EC}_{2}\left(t_{0}, t_{w}\right)=\frac{a c_{0}}{1-\beta}\left[1-e^{-(1-\beta) b t_{0}}\right]+a b e^{-(1-\beta) b t_{0}}\left[c_{w} t_{w}+c_{L} t_{L}-c_{L} t_{w}\right]+c_{p} N+c_{t}\left(t_{0}+t_{w}\right) .
$$

The following assumptions are set:

(B-I) $c_{L}>c_{w}>c_{0}$

(B-II) $b(1-\beta) c_{w} t_{L}>c_{0}$

(B-III) $b(1-\beta)\left[c_{w} t_{w}+c_{L} t_{L}-c_{L} t_{w}\right]>c_{0}$

and let

$$
Q_{2}\left(t_{w}\right)=a b\left[c_{0}-b(1-\beta)\left(c_{w} t_{w}+c_{L} t_{L}-c_{L} t_{w}\right)\right]+c_{t}
$$

Theorem 3.3. Given $c_{0}, c_{w}, c_{L}, c_{p}, c_{t}, N, t_{w}, t_{L}$, the optimal release time $t_{0}^{*}$, which minimizes the total expected software cost $E C_{2}\left(t_{0}, t_{w}\right)$ can be determined as follows based on B-I to B-III.

Case 1. When $Q_{2}\left(t_{w}\right)<0$, a finite and unique $t_{0}^{*}(>0)$ that minimizes the total expected software cost $E C_{2}\left(t_{0}, t_{w}\right)$ exists:

$$
\begin{aligned}
E C_{2}\left(t_{0}^{*}, t_{w}\right)= & \frac{a c_{0}}{1-\beta}\left[1-e^{-(1-\beta) b t_{0}^{*}}\right]+a b e^{-(1-\beta) b t_{0}^{*}}\left[c_{w} t_{w}+c_{L} t_{L}-c_{L} t_{w}\right] \\
& +c_{p} N+c_{t}\left(t_{0}^{*}+t_{w}\right)
\end{aligned}
$$

Case 2. When $Q_{2}\left(t_{w}\right) \geq 0, t_{0}^{*}=0$ and

$$
E C_{2}\left(t_{0}^{*}, t_{w}\right)=a b\left[c_{w} t_{w}+c_{L} t_{L}-c_{L} t_{w}\right]+c_{p} N+c_{t} t_{w} .
$$

The software debugging becomes optimal by only an acceptance test by the user in the operational phase.

Proof. Omitted.

Theorem 3.4. Given $c_{0}, c_{w}, c_{L}, c_{p}, c_{t}, N, t_{0}, t_{L}$, the optimal warranty period $t_{w}^{*}$, which minimizes the total expected software cost $E C_{2}\left(t_{0}, t_{w}\right)$ is determined by the following.

Case 1. When $c_{t} \geq a b e^{-(1-\beta) b t_{0}}\left(c_{L}-c_{w}\right), t_{w}^{*}=0$ minimizes $E C_{2}\left(t_{0}, t_{w}\right)$.

$$
E C_{2}\left(t_{0}, t_{w}^{*}\right)=\frac{a c_{0}}{1-\beta}\left[1-e^{-(1-\beta) b t_{0}}\right]+a b e^{-(1-\beta) b t_{0}} c_{L} t_{L}+c_{p} N+c_{t} t_{0} .
$$

Case 2. When $c_{t} \leq a b e^{-(1-\beta) b t_{0}}\left(c_{L}-c_{w}\right), t_{w}^{*}=t_{L}$ minimizes $E C_{2}\left(t_{0}, t_{w}\right)$.

$$
E C_{2}\left(t_{0}, t_{w}^{*}\right)=\frac{a c_{0}}{1-\beta}\left[1-e^{-(1-\beta) b t_{0}}\right]+a b e^{-(1-\beta) b t_{0}} c_{w} t_{L}+c_{p} N+c_{t}\left(t_{0}+t_{L}\right) .
$$

Proof. Omitted.

The algorithm presented by Rinsaka and Dohi (2006) for simultaneously determining the optimal release time and the optimal warranty period which minimize $\mathrm{EC}_{2}\left(t_{0}, t_{w}\right)$ is similar to previous algorithm. 
Table 4.1. Software failure occurrence time data from System T1

\begin{tabular}{cccccccc}
\hline & \multicolumn{3}{c}{ software failure times (CPUs) } \\
\hline 3 & 1846 & 5324 & 10258 & 15806 & 26770 & 42296 & 56485 \\
13 & 1872 & 5389 & 10491 & 16185 & 27753 & 42296 & 56560 \\
146 & 1986 & 5565 & 10625 & 16229 & 28460 & 45406 & 57042 \\
227 & 2311 & 5623 & 10982 & 16358 & 28493 & 46653 & 62551 \\
342 & 2366 & 6080 & 11175 & 17168 & 29361 & 47596 & 62651 \\
351 & 2608 & 6380 & 11411 & 17458 & 30085 & 48296 & 62661 \\
353 & 2676 & 6477 & 11442 & 17758 & 32408 & 49171 & 63732 \\
444 & 3098 & 6740 & 11811 & 18287 & 35338 & 49416 & 64106 \\
556 & 3278 & 7192 & 12559 & 18568 & 36799 & 50145 & 64893 \\
571 & 3288 & 7447 & 12559 & 18728 & 37642 & 52042 & 71043 \\
709 & 4434 & 7644 & 12791 & 19556 & 37654 & 52489 & 74364 \\
759 & 5036 & 7837 & 13121 & 20567 & 37915 & 52875 & 75409 \\
836 & 5049 & 7843 & 13486 & 21012 & 39715 & 53321 & 76057 \\
860 & 5085 & 7922 & 14708 & 21308 & 40580 & 53443 & 81542 \\
968 & 5089 & 8738 & 15251 & 23063 & 42015 & 54433 & 82702 \\
1056 & 5089 & 10089 & 15261 & 24127 & 42045 & 55381 & 84566 \\
1726 & 5097 & 10237 & 15277 & 25910 & 42188 & 56463 & 88682 \\
\hline
\end{tabular}

Table 4.2. Optimal release time and its corresponding cost for Policy 1

\begin{tabular}{|c|c|c|c|c|c|c|c|c|c|c|}
\hline \multirow{2}{*}{$c_{t}$} & \multicolumn{2}{|c|}{$t_{w}=200$} & \multicolumn{2}{|c|}{$t_{w}=210$} & \multicolumn{2}{|c|}{$t_{w}=220$} & \multicolumn{2}{|c|}{$t_{w}=230$} & \multicolumn{2}{|c|}{$t_{w}=250$} \\
\hline & $t_{0}^{*}$ & $\mathrm{EC}_{1}\left(t_{0}^{*}, t_{w}\right)$ & $t_{0}^{*}$ & $\mathrm{EC}_{1}\left(t_{0}^{*}, t_{w}\right)$ & $t_{0}^{*}$ & $\mathrm{EC}_{1}\left(t_{0}^{*}, t_{w}\right)$ & $t_{0}^{*}$ & $\mathrm{EC}_{1}\left(t_{0}^{*}, t_{w}\right)$ & $t_{0}^{*}$ & $\mathrm{EC}_{1}\left(t_{0}^{*}, t_{w}\right)$ \\
\hline 0.20 & 982.29 & 1815.19 & 923.48 & 1803.87 & 863.58 & 1790.37 & 763.39 & 1772.52 & 628.26 & 1745.74 \\
\hline 0.21 & 752.12 & 1823.45 & 709.19 & 1814 & 624.31 & 1805 & 531.31 & 1785.57 & 389.51 & 1755.21 \\
\hline 0.22 & 562.82 & 1833.28 & 481.53 & 1822.82 & 423.10 & 1813.28 & 333.25 & 1794.82 & 153.12 & 1758.74 \\
\hline 0.23 & 354.14 & 1842.92 & 298.19 & 1830.67 & 216.54 & 1820.12 & 120.51 & 1812.36 & 0 & 1760.63 \\
\hline 0.24 & 152.21 & 1849.11 & 98.28 & 1836.52 & 23.56 & 1826.65 & 0 & 1809.54 & 0 & 1762.86 \\
\hline 0.25 & 0 & 1953.65 & 0 & 1840.88 & 0 & 1829.43 & 0 & 1811.17 & 0 & 1764.64 \\
\hline
\end{tabular}

\section{Numerical Example}

In this chapter, we apply proposed cost models to a set of real testing data of System T1 (Musa, 1979). This system T1 data is summarized as a software failure occurrence time data per fault. Using the maximum likelihood estimate method, the estimates of parameter of Pham (1993) model can be obtained:

$$
\hat{a}=132.6, \quad \hat{b}=3.52 \times 10^{-5}, \quad \hat{\beta}=0.1337 .
$$

The estimated mean value function becomes:

$$
\hat{m}(t)=156.0648\left[1-e^{-0.00003049 t}\right]
$$

We assume the following parameters to obtain optimal release time and warranty period in the proposed cost model as

$$
c_{0}=1.0, \quad c_{w}=3.0, \quad c_{L}=20.0, \quad c_{p}=2.0, \quad t_{L}=1000 \quad \text { and } \quad N=60 .
$$

The optimal release times are then calculated for different values of testing cost per unit time $c_{t}$ and warranty period $t_{w}$. The results are tabulated and shown in Tables 4.2 and 4.3 , respectively.

From Tables 4.2 and 4.3, it can be seen that when the testing cost per unit time $c_{t}$ for fixed the warranty period $t_{w}$ increases, the optimal release time $t_{0}^{*}$ is shorten and the total expected software 
Table 4.3. Optimal release time and its corresponding cost for Policy 2

\begin{tabular}{|c|c|c|c|c|c|c|c|c|c|c|}
\hline \multirow[b]{2}{*}{$c_{t}$} & \multicolumn{2}{|c|}{$t_{w}=200$} & \multicolumn{2}{|c|}{$t_{w}=210$} & \multicolumn{2}{|c|}{$t_{w}=220$} & \multicolumn{2}{|c|}{$t_{w}=230$} & \multicolumn{2}{|c|}{$t_{w}=250$} \\
\hline & $t_{0}^{*}$ & $\mathrm{EC}_{2}\left(t_{0}^{*}, t_{w}\right)$ & $t_{0}^{*}$ & $\mathrm{EC}_{2}\left(t_{0}^{*}, t_{w}\right)$ & $t_{0}^{*}$ & $\mathrm{EC}_{2}\left(t_{0}^{*}, t_{w}\right)$ & $t_{0}^{*}$ & $\mathrm{EC}_{2}\left(t_{0}^{*}, t_{w}\right)$ & $t_{0}^{*}$ & $\mathrm{EC}_{2}\left(t_{0}^{*}, t_{w}\right)$ \\
\hline 0.20 & 1034.35 & 1798.78 & 959.09 & 1785.72 & 882.76 & 1772.45 & 805.28 & 1758.96 & 646.86 & 1731.28 \\
\hline 0.21 & 809.42 & 1809.99 & 734.15 & 1796.28 & 657.80 & 1782 & 580.33 & 1768.18 & 421.91 & 1739.11 \\
\hline 0.22 & 594.94 & 1819.00 & 519.67 & 1804.64 & 443.32 & 179 & 365.86 & 1775.20 & 207.43 & 1744.75 \\
\hline 0.23 & 389.99 & 1825.92 & 314.73 & 1810.91 & 238.38 & 1795.65 & 160.91 & 1780.13 & 2.49 & 1748.29 \\
\hline 0.24 & 193.77 & 1830.83 & 118.51 & 1815.17 & 42.16 & 1799.24 & 0 & 1783.08 & 0 & 1750.92 \\
\hline 0.25 & 5.56 & 1833.82 & 0 & 1817.63 & 0 & 1801.49 & 0 & 1785.38 & 0 & 1753.29 \\
\hline
\end{tabular}

Table 4.4. Optimal warranty period and its corresponding for Policy 1

\begin{tabular}{|c|c|c|c|c|c|c|c|c|c|c|}
\hline \multirow[b]{2}{*}{$c_{t}$} & \multicolumn{2}{|c|}{$t_{0}=500$} & \multicolumn{2}{|c|}{$t_{0}=510$} & \multicolumn{2}{|c|}{$t_{0}=520$} & \multicolumn{2}{|c|}{$t_{0}=530$} & \multicolumn{2}{|c|}{$t_{0}=550$} \\
\hline & $t_{w}^{*}$ & $\mathrm{EC}_{1}\left(t_{0}^{*}, t_{w}\right)$ & $t_{w}^{*}$ & $\mathrm{EC}_{1}\left(t_{0}^{*}, t_{w}\right)$ & $t_{w}^{*}$ & $\mathrm{EC}_{1}\left(t_{0}^{*}, t_{w}\right)$ & $t_{w}^{*}$ & $\mathrm{EC}_{1}\left(t_{0}^{*}, t_{w}\right)$ & $t_{w}^{*}$ & $\mathrm{EC}_{1}\left(t_{0}^{*}, t_{w}\right)$ \\
\hline 0.20 & 387.50 & 3104.15 & 377.50 & 3117.76 & 367.50 & 3131.34 & 357.50 & 3144.89 & 337.50 & 3171.90 \\
\hline 0.21 & 357.06 & 3115.88 & 17.06 & 3129.48 & 37.06 & 3143 & 327.06 & 315 & 307.06 & 3183.62 \\
\hline 0.22 & 326.83 & 3127.30 & 316.83 & 3140.90 & 306.83 & 3154.48 & 296.83 & 3168.03 & 276.83 & 3195.04 \\
\hline 0.23 & 296.80 & 3138.41 & 286.80 & 3152.02 & 276.80 & 3165.60 & 266.80 & 3179.15 & 246.80 & 3206.16 \\
\hline 0.24 & 266.95 & 3149.23 & 256.95 & 3162.84 & 246.95 & 3176.97 & 236.95 & 3189.97 & 216.95 & 3121.98 \\
\hline 0.25 & 237.31 & 3165.75 & 227.31 & 3173.36 & 217.31 & 3186.94 & 207.31 & 3200.49 & 187.31 & 3227.50 \\
\hline
\end{tabular}

Table 4.5. Optimal warranty period and its corresponding for Policy 2

\begin{tabular}{|c|c|c|c|c|c|c|c|c|c|c|}
\hline \multirow{2}{*}{$c_{t}$} & \multicolumn{2}{|c|}{$t_{0}=500$} & \multicolumn{2}{|c|}{$t_{0}=510$} & \multicolumn{2}{|c|}{$t_{0}=520$} & \multicolumn{2}{|c|}{$t_{0}=530$} & \multicolumn{2}{|c|}{$t_{0}=550$} \\
\hline & $t_{w}^{*}$ & $\mathrm{EC}_{2}\left(t_{0}^{*}, t_{w}\right)$ & $t_{w}^{*}$ & $\mathrm{EC}_{2}\left(t_{0}^{*}, t_{w}\right)$ & $t_{w}^{*}$ & $\mathrm{EC}_{2}\left(t_{0}^{*}, t_{w}\right)$ & $t_{w}^{*}$ & $\mathrm{EC}_{2}\left(t_{0}^{*}, t_{w}\right)$ & $t_{w}^{*}$ & $\mathrm{EC}_{2}\left(t_{0}^{*}, t_{w}\right)$ \\
\hline 0.20 & 1000 & 763.774 & 1000 & 766.134 & 1000 & 768.493 & 1000 & 770.851 & 1000 & 775.566 \\
\hline 0.21 & 1000 & 778.774 & 1000 & 783.234 & 1000 & 783.693 & 1000 & 786.151 & 1000 & 791.066 \\
\hline 0.22 & 1000 & 793.774 & 1000 & 796.893 & 1000 & 798.893 & 1000 & 801.451 & 1000 & 806.566 \\
\hline 0.23 & 1000 & 808.774 & 1000 & 811.434 & 1000 & 814.093 & 1000 & 816.751 & 1000 & 822.066 \\
\hline 0.24 & 1000 & 823.774 & 1000 & 826.534 & 1000 & 829.293 & 1000 & 832.051 & 1000 & 837.566 \\
\hline 0.25 & 1000 & 838.774 & 1000 & 841.634 & 1000 & 844.493 & 1000 & 847.351 & 1000 & 853.066 \\
\hline
\end{tabular}

Table 4.6. Optimal release time and warranty period for Policy 1 and 2

\begin{tabular}{|c|c|c|c|c|c|c|}
\hline \multirow[b]{2}{*}{$c_{t}$} & \multicolumn{3}{|c|}{ Policy 1} & \multicolumn{3}{|c|}{ Policy 2} \\
\hline & $t_{0}^{* *}$ & $t_{w}^{* *}$ & $\mathrm{EC}_{1}\left(t_{0}^{* *}, t_{w}^{* *}\right)$ & $t_{0}^{* *}$ & $t_{w}^{* *}$ & $\mathrm{EC}_{2}\left(t_{0}^{* *}, t_{w}^{* *}\right)$ \\
\hline 0.20 & 237.48 & 882.37 & 678.89 & 375.55 & 864.32 & 645.67 \\
\hline 0.21 & 108.22 & 1000 & 689.10 & 246.75 & 962.89 & 688.39 \\
\hline 0.22 & 0 & 1000 & 697.37 & 113.47 & 1000 & 707.35 \\
\hline 0.23 & 0 & 1000 & 702.48 & 0 & 1000 & 723.54 \\
\hline 0.24 & 0 & 1000 & 706.12 & 0 & 1000 & 737.75 \\
\hline 0.25 & 0 & 1000 & 703.31 & 0 & 1000 & 753.56 \\
\hline
\end{tabular}

cost increases. In addition, when the warranty period $t_{w}$ for fixed the testing cost per unit time $c_{t}$ increases, the optimal release time decreases. Comparing Policy 1 and Policy 2, the optimal release time of Policy 1 is always earlier than that of Policy 2 and the total expected software cost of Policy 1 for optimal release time is always later than that of Policy 2; this is because the reliability increases and the residual faults in the software were effectively detected and removed in the operational phase during a given warranty period.

Table 4.4 and 4.5 examine the effect of the testing cost per unit time $c_{t}$ and release time $t_{0}$ on the optimal warranty period $t_{w}^{*}$ for the proposed Policy 1 and 2. It can be seen that when the testing cost per unit time $c_{t}$ for fixed the release time $t_{0}$ increases, the optimal warranty period $t_{w}^{*}$ shorten and the total expected software cost increases. In addition, when the release time $t_{0}$ for fixed the testing cost per unit time $c_{t}$ increases, the optimal warranty period decreases. Comparing Policy 1 and Policy 2, the optimal warranty period of Policy 1 is always shorter than that of Policy 2 and the total expected software cost of Policy 1 for optimal warranty period is always greater than that of Policy 2; The reason is same in the case determination of the optimal release time. 
Table 4.6 shows the effect of the testing cost per unit time $c_{t}$ on the joint optimal policy of the release time and the warranty period. From Table 4.6, as $c_{t}$ increases, the optimal release time $t_{0}^{* *}$ happens earlier. However, the optimal warranty period $t_{0}^{* *}$ cannot be seen. In addition, as the testing cost per unit time $c_{t}$ increases, $\mathrm{EC}_{1}\left(t_{0}^{* *}, t_{w}^{* *}\right), \mathrm{EC}_{2}\left(t_{0}^{* *}, t_{w}^{* *}\right)$ can be confirmed to also decrease.

\section{Conclusions}

This paper defined the time support by the project team ends in the operational phase as the warranty period and modeled the differences in the software execution environments in the testing phase and the operational phase by considering the software operational profile in the software reliability model based on NHPP. In addition, we considered the problem of determining the optimal testing period and the optimal warranty period that minimize the total expected software cost in terms of periodic software maintenance (e.g. update, service pack, etc). As a future problem, the same model can be built and analyzed even in other periodic software maintenance (e.g. upgrade, version up) policies.

\section{References}

Cha, J. H. (2000). On a better burn-in procedure, Journal of Applied Probability, 37, 1099-1103.

Cha, J. H. (2001). Burn-in procedures for a generalized model, Journal of Applied Probability, 38, $542-553$.

Dohi, T., Okamura, H., Kaio, N. and Osaki, S. (2000). The age-dependent optimal warranty policy and its application to software maintenance contract, Proceeding 5th International on Probability Safety Assessment Manage, 4, 2547-2552.

Goel, A. L. and Okumoto, K. (1979). Time-dependent error-detection rate model for software reliability and other performance measures, IEEE Transactions on Reliability, R-28, 206-211.

Jelinski, Z. and Moranda, P. B. (1972). Software Reliability Research, Statistical Computer Performance Evaluation, Freiberger, W. Ed., Academic Press, New York.

Kimura, M., Toyota, T. and Yamada, S. (1999). Economic analysis of software release problem with warranty cost and reliability requirement, Reliability Engineering and System Safety, 66, 49-55.

Koch, H. S. and Kubat, P. (1983). Optimal release time of computer software, IEEE Transaction on Software Engineering, 9, 323-327.

Mi, J. (1994). Burn-in and maintenance policies, Advances in Applied Probability, 26, $207-221$.

Musa, J. D. (1979). Software Reliability Data, Technique Report, Roma Air Development Center, USFA.

Okumoto, K. and Goel, A. L. (1980). Optimum release time for software system and based on reliability and cost criteria, Journal of System and Software, 1, 315-318.

Pham, H. (1993). Software reliability assessment: Imperfect debugging and multiple failure types in software development, EG\&G-RAMM-10737, Idaho National Engineering Laboratory.

Pham, H. and Zhang, X. (1999). A software cost model with warranty and risk costs, IEEE Transactions on Computers, 48, 71-75.

Rinsaka, K. and Sandoh, H. (1999). A study on software maintenance service contracts, Transactions of IEICE, J82-A, 1819-1829.

Rinsaka, K. and Dohi, T. (2006). Optimal testing/maintenance design in a software development project, Electronics and Communications in Japan, 89, 953-961.

Yamada, S. (1994). Optimal release problems with warranty period based on a software maintenance cost model, Transactions on IPS Japan, 35, 2197-2202. 